\title{
Induced sclerotium formation exposes new bioactive metabolites from Aspergillus sclerotiicarbonarius
}

\author{
Lene M Petersen ${ }^{1}$, Jens C Frisvad ${ }^{1}$, Peter B Knudsen ${ }^{1}$, Marko Rohlfs ${ }^{2}$, Charlotte H Gotfredsen ${ }^{3}$ and \\ Thomas O Larsen ${ }^{1}$
}

Sclerotia are known to be fungal survival structures, and induction of sclerotia may prompt production of otherwise undiscovered metabolites. Aspergillus sclerotiicarbonarius (IBT 28362) was investigated under sclerotium producing conditions, which revealed a highly altered metabolic profile. Four new compounds were isolated from cultivation under sclerotium formation conditions and their structures elucidated using different analytical techniques (HRMS, UV, 1D and 2D NMR). This included sclerolizine, an alkylated and oxidized pyrrolizine, the new emindole analog emindole SC and two new carbonarins; carbonarins I and J. We have identified the three latter as true sclerotial metabolites. All metabolites were tested for antifungal and antiinsectan activity, and sclerolizine and carbonarin I displayed antifungal activity against Candida albicans, while all four showed antiinsectan activity. These results demonstrate induction of sclerotia as an alternative way of triggering otherwise silent biosynthetic pathways in filamentous fungi for the discovery of novel bioactive secondary metabolites.

The Journal of Antibiotics (2015) 68, 603-608; doi:10.1038/ja.2015.40; published online 6 May 2015

\section{INTRODUCTION}

Natural products and their derivatives have historically been widely used as a source of therapeutic agents and among these are secondary metabolites (SMs) produced by filamentous fungi. In general, SMs represent a wide array of structural diversity and display a broad range of biological activities, which can be utilized for pharmaceutical purposes.

The classical approach for natural product discovery has typically involved screening of crude extracts from various natural sources followed by purification and structural elucidation. With recent advances in genome sequencing projects, it has however become evident that several microorganisms have a far greater biosynthetic potential and metabolic diversity than previously assumed. To discover all metabolites that an organism is capable of producing, several approaches can be taken such as the OSMAC (One Strain Many Compounds), ${ }^{1}$ use of epi-genetic inducers, ${ }^{2}$ co-culturing experiments ${ }^{3}$ or various methods within genome mining. $^{2}$

We have recently reported conditions triggering sclerotium production in filamentous fungi, ${ }^{4}$ where we demonstrated that under these circumstances a great alteration in the chemical profile can be achieved. ${ }^{4,5}$ In this study, we follow up and show that the metabolic profile of A. sclerotiicarbonarius (IBT 28362) is greatly altered, when sclerotia are produced, leading to the discovery of four novel compounds.

\section{MATERIALS AND METHODS}

Media

The CYA media used were prepared as described by Frisvad and Samson. ${ }^{6}$ To trigger sclerotia production, raisins were added to the media (CYAR media) as described by Frisvad et $a l^{4}$

Fungal growth and extraction

Plates were incubated at $25^{\circ} \mathrm{C}$ for 7 days in the dark. For standard screening, 3-5 plugs were taken from one colony by use of a 6-mm plug drill, covering the diameter of one colony and extracted by the micro-scale extraction method described by Smedsgaard. ${ }^{7}$ Sclerotia were harvested and extracted as described by Frisvad et al. ${ }^{4}$

Chemical analysis of strains

Analysis was performed using reversed phase ultra-HPLC (UPHLC) $\mathrm{UV} / \mathrm{Vis}$ diode array detector (DAD) high-resolution time-of-flight mass spectrometer on a maXis G3 orthogonal acceleration quadrupole time of flight (Q-TOF) mass spectrometer (Bruker Daltonics, Bremen, Germany) as described by Holm et al. ${ }^{8}$

Purification of metabolites

A. sclerotiicarbonarius (IBT 28362=CBS 121057) was inoculated as three point inoculations on 112 plates of CYAR medium and incubated at $25^{\circ} \mathrm{C}$ for 7 days in the dark. The plates were harvested and extracted twice overnight with ethyl acetate with $1 \%$ formic acid

\footnotetext{
${ }^{1}$ Natural Product Discovery Group, Department of Systems Biology, Technical University of Denmark, Lyngby, Denmark; ${ }^{2}$ Blumenbach Institute of Zoology and Anthropology, Animal Ecology, Georg-August-University of Göttingen, Göttingen, Germany and ${ }^{3}$ Department of Chemistry, Technical University of Denmark, Lyngby, Denmark Correspondence: Dr TO Larsen, Natural Product Discovery Group, Department of Systems Biology, Technical University of Denmark, Soeltofts Plads B221, 2800 Kgs. Lyngby, Denmark.

E-mail: tol@bio.dtu.dk

Received 29 January 2015; revised 9 March 2015; accepted 16 March 2015; published online 6 May 2015
} 


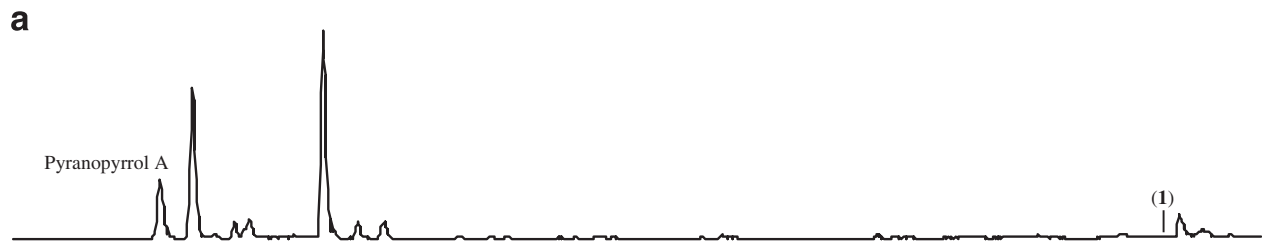

b
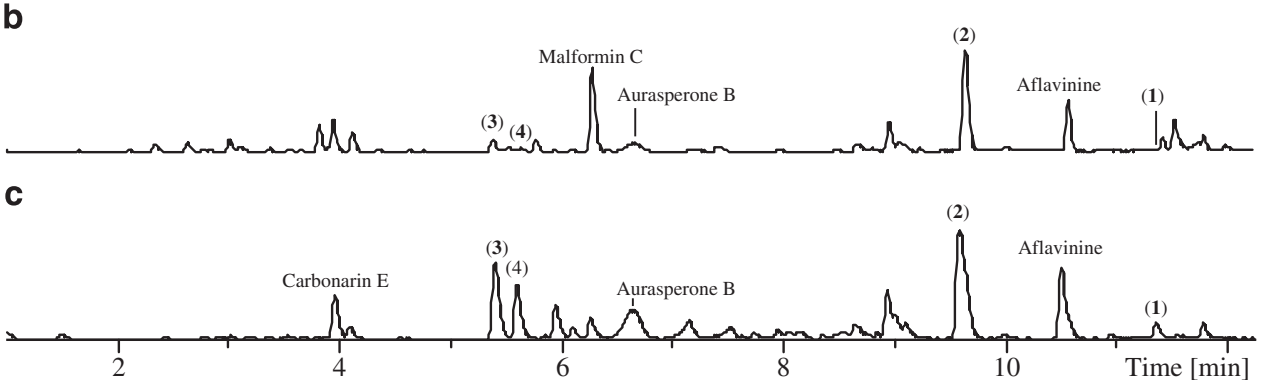

Figure 1 UHPLC-TOF-HRMS ESI+BPC chromatogram of Aspergillus sclerotiicarbonarius (IBT 28362) extractions. Scaled to RT between 1 and 12 min, intensities are to scale. (a) Plug extraction from growth with no sclerotium production. (b) Plug extraction from growth with sclerotium production. (c) Sclerotial extraction. The extractions under sclerotium production $(\mathbf{b}+\mathbf{c})$ show the presence of the four novel compounds sclerolizine (1), emindole SC (2), carbonarin I (3) and carbonarin J (4).

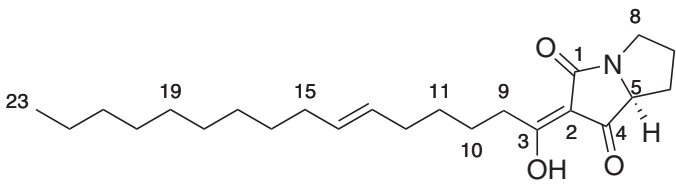

Sclerolizine (1)<smiles>COc1cc(OC)c2c(O)c3c(c(-c4cc(C5=C(Cc6ccc(O)c(OC)c6)CNC5=O)ccc4O)c2c1)OC(C)(O)CC3=O</smiles>

Carbonarin I (3)

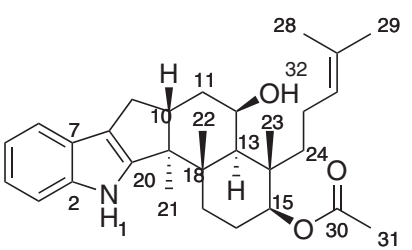

Emindole SC (2)<smiles>COc1cc(O)cc(CC2=C(c3ccc(O)c(-c4c5cc(OC)cc(OC)c5c(O)c5c(=O)cc(C)oc45)c3)C(=O)NC2)c1</smiles>

Carbonarin J (4)

Figure 2 Structures of the novel compounds isolated from A. sclerotiicarbonarius.

and work-up was performed as described by Holm et al. ${ }^{8}$ The extract was fractionated using an Isolera flash purification system (Biotage, Uppsala, Sweden) by adsorption onto $\mathrm{C}_{18}$ column material and packing into a SNAP column (Biotage) with $\mathrm{C}_{18}$ material. The extract was then fractionated using a linear gradient of acetonitrile-water starting at $30 \%$ acetonitrile and increasing to $100 \%$. Acetonitrile used was of HPLC grade and $\mathrm{H}_{2} \mathrm{O}$ was purified and deionized by a Millipore system through a $0.22-\mu \mathrm{m}$ membrane filter (milliQ-water). The flow rate used was $40 \mathrm{ml}$ per minute, the column volume $124 \mathrm{ml}$, and fractions were collected automatically one column volume at a time.

The Isolera fractions were subjected to further purification on a semi-preparative HPLC which was either a Waters 600 Controller with a 996 photodiode array detector (Waters, Milford, MA, USA) or a Gilson 322 Controller connected to a 215 Liquid Handler, 819 Injection Module and a $172 \mathrm{DAD}$ (Gilson, Middleton, WI, USA), This was achieved using a Luna II $\mathrm{C}_{18}$ column $(250 \times 10 \mathrm{~mm}, 5 \mu \mathrm{m}$, 
Table $1{ }^{1} \mathrm{H}(500 \mathrm{MHz})$ and ${ }^{13} \mathrm{C}(125 / 100 \mathrm{MHz})$ NMR spectroscopic data for compounds $1-4$

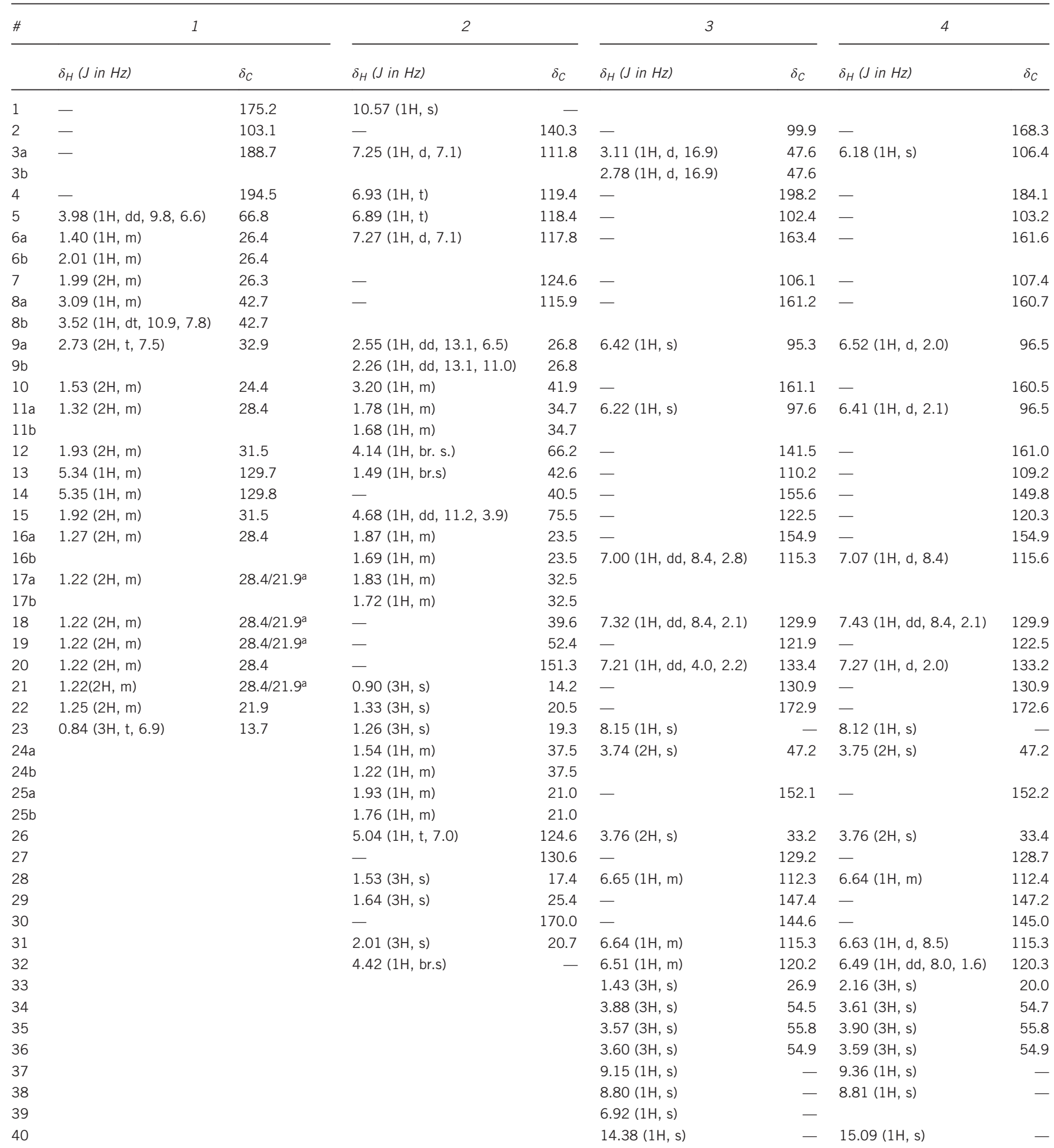

aDe to overlapping signals the specific carbon chemical shift could not be determined. For compounds $\mathbf{1}$ and $\mathbf{2}$, the ${ }^{13} \mathrm{C}$-NMR chemical shifts were determined from HSQC and HMBC experiments.

Phenomenex) or a Gemini C6-Phenyl 110A column $(250 \times 10.00 \mathrm{~mm}$, $5 \mu \mathrm{m}$, Phenomenex, Torrance, CA, USA). In all, $50 \mathrm{ppm}$ trifluoroacetic acid was added to acetonitrile of HPLC grade and milliQ-water. For choice of system, flow rate, column, gradients and yields see Supplementary information S23. Spectroscopic data for all compounds are given in the Supplementary information.

\section{NMR and structural elucidation}

${ }^{1} \mathrm{H}$ and $2 \mathrm{D}$ spectra were recorded on a Varian Unity Inova-500 $\mathrm{MHz}$ spectrometer (Varian, Palo Alto, CA, USA), while ${ }^{13} \mathrm{C}$ spectra were recorded on a Bruker Ascend $400 \mathrm{MHz}$ spectrometer (Bruker, Bremen, Germany). Spectra were acquired using standard pulse sequences. The deuterated solvent was DMSO- $d_{6}$ and signals were 
referenced to the solvent signals for DMSO- $d_{6}$ at $\delta_{\mathrm{H}}=2.49 \mathrm{ppm}$ and $\delta_{\mathrm{C}}=39.5 \mathrm{ppm}$. The NMR data were processed in MestReNova v.6.0.15391. Chemical shifts are in ppm $(\delta)$ and scalar couplings are reported in hertz $(\mathrm{Hz})$. Optical rotation was measured on a Perkin-Elmer 321 Polarimeter (Perkin Elmer, Waltham, MA, USA).

\section{Antifungal susceptibility testing}

All compounds were screened for antifungal activity toward Candida albicans in accordance with the CLSI standards, as described by Holm et al. ${ }^{8}$

\section{Drosophila larval testing}

All compounds were screened for larvicidal activity toward Drosophila melanogaster larvae as described by Trienens et al., ${ }^{9}$ but using $\mathrm{MeOH}$ as a solvent.

\section{RESULTS AND DISCUSSION}

The metabolic profile of A. sclerotiicarbonarius (IBT 28362) was investigated under conditions both with and without conditions favoring sclerotium formation. The latter was achieved using media containing raisins (CYAR media), as earlier described. ${ }^{4}$ Without the presence of sclerotia, the metabolic profile (Figure 1a) displayed only few metabolites. One of the most abundant metabolites, pyranonigrin A (pyranopyrrol A) (verified with UHPLC-DAD-HRMS by comparison of retention time, monoisotopic mass for the pseudomolecular ion, adducts and UV spectrum with an authentic standard), is known from other black aspergilli. ${ }^{10,11}$ With formation of sclerotia, a more diverse production of SMs was observed, both in a mycelium plug extraction and by true sclerotial extraction (Figure $1 \mathrm{~b}$ and $\mathrm{c}$ ). Known black aspergilli SMs such as aurasperone $\mathrm{B}^{12}$ and malformin $\mathrm{C}^{13}$ were detected (verified by UHPLC-DAD-HRMS). In our previous studies, we observed several aflavinines in the sclerotial extracts, and production of aurasperone B as a common trend, ${ }^{4}$ which was indeed also the case for A. sclerotiicarbonarius.

From the sclerotial extract, several putative unknown compounds were detected. From a larger extract cultivated under sclerotium formation conditions, these compounds were purified and their structures solved by 1D and 2D NMR spectroscopy, Figure 2 and Table 1.

\section{Structural elucidation of four new compounds}

The UV spectrum of sclerolizine displayed absorption maxima at $280 \mathrm{~nm}$, and the ESI+ HRMS spectrum displayed one adduct, with $\mathrm{m} /$ $z=376.2855$, from which the molecular formula could be deduced to $\mathrm{C}_{23} \mathrm{H}_{37} \mathrm{NO}_{3}$ (376.2846 calculated for $\left[\mathrm{C}_{23} \mathrm{H}_{37} \mathrm{NO}_{3}+\mathrm{H}\right]^{+}$). The ${ }^{1} \mathrm{H}$ NMR spectra revealed the presence of one $\mathrm{H}_{\alpha}$ proton (H5), which was found to be part of a COSY spin system with three methylene groups (C6, C7 and C8), which altogether were elucidated as the pyrrolidine ring. The remaining resonances in the ${ }^{1} \mathrm{H}$ NMR spectra belonged to a larger spin system with two overlapping protons in the double bond area, 1 methyl and 12 methylene groups, whereof five ( $\mathrm{H} 17, \mathrm{H} 18, \mathrm{H} 19, \mathrm{H} 20$ and $\mathrm{H} 21$ ) were overlapping at $\delta_{\mathrm{H}} 1.22 \mathrm{ppm}$. The linking between the two COSY spin systems and the assignment

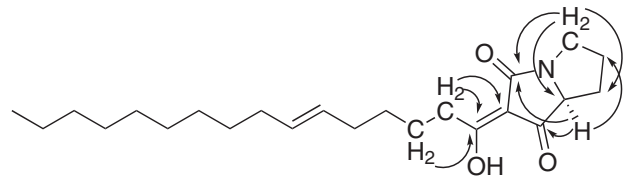

Figure 3 Key HMBC correlations of sclerolizine (1). of four quaternary carbons (two carbonyls and two double bond carbons) were accomplished through detailed analysis of the HMBC spectral data (Figure 3). It is noted that there was no HMBC correlations linking the two spin systems, but to justify the chemical shifts observed, the suggested structure was the only appropriate proposal. The resonances originating from the C13-C14 double bond were very close in chemical shift leading to a second-order spin system. Consequently, it was not possible to accurately determine the size of the $J$ coupling constant. A value of $J$ could however be measured to $15.2 \mathrm{~Hz}$ in the second-order system, suggesting a trans double bond. Furthermore, the chemical shifts for the neighboring allylic carbons at $31.5 \mathrm{ppm}$ (C12 and $\mathrm{C} 15)$ were also in consistence with a trans configuration as allylic carbons in cis fatty acids would be further up field, typically at values below $30 \mathrm{ppm} .{ }^{14}$ The stereochemistry of the C2-C3 double bond and C5 has not been investigated, but we hypothesize that L-proline is incorporated into the molecule. Similar synthetic structures have been patented, ${ }^{15,16}$ however to the best of our knowledge, this is the first report of an oxidized pyrrolizine from a natural source.

The UV spectrum of emindole SC displayed absorption maxima at $283 \mathrm{~nm}$, and the ESI+ HRMS spectrum displayed one adduct, with $\mathrm{m} /$ $z=464.3164$, from which the molecular formula could be deduced to $\mathrm{C}_{30} \mathrm{H}_{41} \mathrm{NO}_{3}$ (464.3159 calculated for $\left.\left[\mathrm{C}_{30} \mathrm{H}_{41} \mathrm{NO}_{3}+\mathrm{H}\right]^{+}\right)$. The backbone of emindole SC displays the same skeleton as emindole SB from Emericella striata. ${ }^{17}$ The main difference of emindole SC is an extra hydroxyl group $(\mathrm{C} 12)$ and an acetylation at $\mathrm{C} 15$. This gives rise to additional signals in the ${ }^{1} \mathrm{H}$ NMR spectrum from the methyl group $\mathrm{C} 31$, and $\mathrm{HMBC}$ correlations to the carbonyl at $\delta_{\mathrm{C}} 170 \mathrm{ppm}(\mathrm{C} 30)$ from H15 and H31. The chemical shift of H12 is shifted downfield to $\delta_{\mathrm{H}} 4.14 \mathrm{ppm}$, and the addition of the hydroxyl is also evident from the carbon chemical shift, which is moved downfield to $\delta_{\mathrm{C}} 66.2 \mathrm{ppm}$. Otherwise, the NMR data are very comparable to those of emindole SB. ${ }^{17}$ NOESY experiments enabled determination of the relative stereochemistry for the stereogenic centers. NOE connectivities were found from $\mathrm{H} 13$ to $\mathrm{H} 12, \mathrm{H} 15$ and $\mathrm{H} 21$ placing these on the same side of the ring systems, which is also observed for other emindoles. This was further verified by strong NOESY connectivities between $\mathrm{H} 10$ and $\mathrm{H} 22$ as well as $\mathrm{H} 23$ and $\mathrm{H} 32$.

The UV spectra of carbonarins I and J displayed absorption maxima at $410 \mathrm{~nm}$ suggesting a highly conjugated chromophore. Analysis of the HRMS data showed $\mathrm{m} / \mathrm{z}$ of 614.2025 and 596.1923, which was used to determine the molecular formula to $\mathrm{C}_{34} \mathrm{H}_{31} \mathrm{NO}_{10}$ and $\mathrm{C}_{34} \mathrm{H}_{29} \mathrm{NO}_{9}$ (614.2021 and 596.1915 calculated for $\left[\mathrm{C}_{34} \mathrm{H}_{31} \mathrm{NO}_{10}+\mathrm{H}\right]$ + and $\left[\mathrm{C}_{34} \mathrm{H}_{29} \mathrm{NO}_{9}+\mathrm{H}\right]^{+}$, respectively).

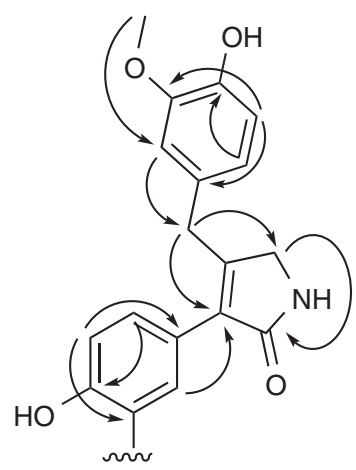

Figure 4 Key HMBC correlations of the upper part of carbonarins I (3) and J (4). 
Table 2 Presence of compounds 1-4 with and without sclerotium production

\begin{tabular}{llll}
\hline & $\begin{array}{l}\text { Plug extraction CYA } \\
\text { (no sclerotium formation) }\end{array}$ & $\begin{array}{l}\text { Plug extraction CYA } \\
\text { (sclerotium formation) }\end{array}$ & $\begin{array}{l}\text { Sclerotial } \\
\text { extraction }\end{array}$ \\
\hline Sclerolizine (1) & $\mathrm{x}$ & $\mathrm{x}$ & $\mathrm{x}$ \\
Emindole SC (2) & - & $\mathrm{x}$ & $\mathrm{x}$ \\
Carbonarin I (3) & - & $\mathrm{x}$ & $\mathrm{x}$ \\
Carbonarin J (4) & - & $\mathrm{x}$ & $\mathrm{x}$ \\
\hline
\end{tabular}

Detected by extracted ion chromatograms with a mass tolerance of $\mathrm{m} / \mathrm{z} \pm 0.005$

Table 3 Antifungal activities of compounds 1-4 against Candida albicans

\begin{tabular}{llc}
\hline Compound & Origin & I $C_{50}(\mu \boldsymbol{M})$ \\
\hline Sclerolizine (1) & A. sclerotiicarbonarius & $8.5 \pm 2.0$ \\
Emindole SC (2) & A. sclerotiicarbonarius & $>100$ \\
Carbonarin I (3) & A. sclerotiicarbonarius & $85.8 \pm 13.1$ \\
Carbonarin J (4) & A. sclerotiicarbonarius & $>100$ \\
\hline
\end{tabular}

Structural elucidation of the two related compounds revealed structures similar to those of carbonarins $\mathrm{A}-\mathrm{H}$, which are metabolites from A. carbonarius. ${ }^{18,19}$ The NMR data revealed a three ring system polyketide part present in both structures, similar to that in other naphthopyrones such as aurasperone $\mathrm{B}^{12}$ and fonsecin $\mathrm{B}^{20}$ (the lower part of the carbonarins, see Figure 2). The ring system is characterized by having only few proton resonances $\left(\mathrm{C}_{16} \mathrm{H}_{15} \mathrm{O}_{6}\right.$ and $\mathrm{C}_{16} \mathrm{H}_{13} \mathrm{O}_{5}$, respectively, for the three ring system in carbonarins $\mathrm{I}$ and $\mathrm{J}$ ). The NMR data for this part of the structure are comparable to that reported for similar structures. ${ }^{20}$ Due to strong intramolecular bonding from the carbonyl group (C4), to the phenolic proton (H40) present in both structures, the latter were resonating at low fields and gave rise to signals at 14.38 and $15.09 \mathrm{ppm}$, respectively. These have also been observed in similar structures. ${ }^{20,21}$ Carbonarins I and J differ only in this part of the structure and only at C2 and C3, with a single bond in the former and a double bond in the latter. C2 of carbonarin I has furthermore a hydroxyl group, shifting the carbon chemical shift dramatically downfield to $\delta_{\mathrm{C}} 99.9 \mathrm{ppm}$. No HMBC correlations were observed to the carbons at $\mathrm{C} 12$ for carbonarin I and $\mathrm{C} 4, \mathrm{C} 12$ or $\mathrm{C} 14$ for carbonarin J, wherefore these were assigned from resonances in the $1 \mathrm{D}{ }^{13} \mathrm{C}$ carbon spectrum.

The upper part of carbonarins I and J is alike (Figures 2 and 4), including two partly substituted aromatic rings and a five membered lactam ring. This part is unique for carbonarins $\mathrm{I}$ and $\mathrm{J}$, even compared with other carbonarins, which show different substitution patterns in the aromatic rings. The NMR data for this part are highly similar for carbonarins I and J, all through C15-C33 and H36-H38, for proton as well as carbon chemical shifts. For this part of the carbonarins, both ${ }^{1} \mathrm{H}$ NMR spectra displayed one amide proton (H23), two phenolic protons ( $\mathrm{H} 37$ and $\mathrm{H} 38)$, six aromatic protons, equally divided on the two aromatic rings (H17, H18, H20, and H28, $\mathrm{H} 31, \mathrm{H} 32$ ), two methylenes ( $\mathrm{H} 24$ and $\mathrm{H} 26$ ) both appearing as singlets, and one further singlet arising from the protons of the O-methyl group (H36). The linking of the spin systems and final assignment were accomplished through detailed analysis of the HMBC and NOESY spectral data. The important HMBC correlations for the structural elucidations of the unique upper part of the carbonarins are

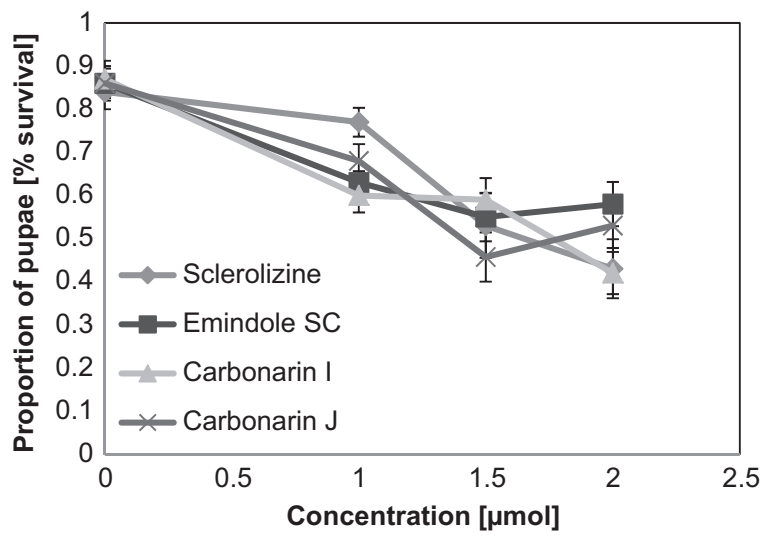

Figure 5 Mean proportional survival of Drosophila larvae to the pupal stage as a function of different concentrations $(\mu \mathrm{M})$ of the $A$. sclerotiicarbonarius new compounds in the insect's food. Error bars depict the standard error. A full color version of this figure is available at The Journal of Antibiotics journal online.

depicted in Figure 4. Linking between this upper part to the three ring polyketide part was supported by HMBC correlations from $\mathrm{H} 20$ to C13 in both carbonarins I and J.

The bottom part consisting of a three ring system polyketide part present in both carbonarins I and J was similar to what is seen in the naphthopyrones fonsecin B. ${ }^{20}$ By comparison with an authentic standard, we detected trace amounts of fonsecin B being produced under conditions with sclerotium formation (data not shown), why we hypothesize that fonsecin B is the likely precursor of the new carbonarins described here.

A further search for carbonarins A-H in the UHPLC-UV-HRMS data from the sclerotial extract showed trace amounts of carbonarins A-D, high production of carbonarins $\mathrm{E}$ and $\mathrm{F}$, while neither carbonarin $\mathrm{G}$ nor $\mathrm{H}$ could be detected (tentatively identified by HRMS with a mass tolerance of $m / z \pm 0.005$ ). An equivalent search for carbonarins in the A. sclerotiicarbonarius extract with no sclerotium production showed no traces of any of the carbonarins, clearly indicatiing that this group of compounds is related to sclerotium production.

Three of the new compounds are true sclerotial metabolites For verification of whether the compounds are true sclerotial metabolites, extracted ion chromatograms with a mass tolerance of \pm 0.005 were performed based on plug extraction from growth conditions with and without sclerotium formation as well as the sclerotium extraction, Table 2 and Supplementary information. It was here found that all metabolites (1-4) were present in both the sclerotial extracts and the plug extracts under sclerotium formation. In the plug extraction where no sclerotia were formed, all compounds, but sclerolizine was absent, strongly indicating that emindole SC, carbonarin I and carbonarin J are true sclerotium metabolites.

\section{Bioactivities}

Compounds 1-4 were tested for antifungal and insecticidal activity. The half maximal $\mathrm{IC}\left(\mathrm{IC}_{50}\right)$ for Candida albicans treated with compounds 1-4 are displayed in Table 3. The $\mathrm{IC}_{50}$ values were calculated based on duplicate experiments carried out in at least two independent trials and annotated with their respective standard deviation. Sclerolizine and carbonarin I displayed moderate antifungal activity, with sclerolizine as the most active. 
As sclerotial compounds have often been found to possess insecticidal properties, ${ }^{22}$ all compounds were furthermore tested for larvicial activity toward Drosophila melanogaster larvae. The results are depicted in Figure 5. With increasing concentrations, all compounds were affecting the survival of the insect larvae $(P<0.001)$, yet there was no compound-specific variation in this effect. Overall, at a concentration of $1.83 \pm 0.08 \mu \mathrm{mol}, 50 \%$ of the larvae were found dead.

\section{CONCLUSION}

We here show that the chemical profile of $A$. sclerotiicarbonarius is greatly altered when cultivated under conditions favoring production of sclerotia. By cultivation of a large-scale extract under these conditions we have isolated four novel SMs of mixed biosynthetic origin and elucidated their structures leading to the new compounds sclerolizine, emindole SC, carbonarins I and J. Our analysis has shown that the three latter compounds were locally restricted to the sclerotia, which indicates that these are true sclerotial metabolites.

Sclerolizine and carbonarin I displayed antifungal activity against Candida albicans and all four compounds antiinsectan activity. This study thus provides an alternative way of triggering silent biosynthetic pathways in filamentous fungi for the discovery of novel bioactive SMs. With the discovery of a perfect state in Aspergillus sclerotiicarbonarius, ${ }^{23}$ further SMs may be discovered in the progeny of this species.

\section{ACKNOWLEDGEMENTS}

This work was supported by grant 09-064967 from the Danish Council for Independent Research, Technology and Production Sciences.

1 Bode, H., Bethe, B., Höfs, R. \& Zeeck, A. Big effects from small changes: possible ways to explore nature's chemical diversity. ChemBioChem 3, 619-627 (2002).

2 Brakhage, A. A. \& Schroeckh, V. Fungal secondary metabolites - strategies to activate silent gene clusters. Fungal Genet. Biol. 48, 15-22 (2011).

3 Schroeckh, V. et al. Intimate bacterial-fungal interaction triggers biosynthesis of archetypal polyketides in Aspergillus nidulans. Proc. Natl Acad. Sci. USA 106, 14558-14563 (2009).
4 Frisvad, J. C., Petersen, L. M., Lyhne, E. K. \& Larsen, T. O. Formation of sclerotia and production of indoloterpenes by Aspergillus niger and other species in section Nigri. PLOS ONE 9, e94857 (2014).

5 Petersen, L. M., Hoeck, C., Frisvad, J. C., Gotfredsen, C. H. \& Larsen, T. O. Dereplication guided discovery of secondary metabolites of mixed biosynthetic origin from Aspergillus aculeatus. Molecules 19, 10898-10921 (2014).

6 Samson, R. A., Houbraken, J., Thrane, U., Frisvad, J. C. \& Andersen, B. Food and Indoor Fungi (CBS-KNAW Fungal Biodiversity Centre, Utrecht, The Netherlands, 2010).

7 Smedsgaard, J. Micro-scale extraction procedure for standardized screening of fungal metabolite production in cultures. J. Chromatogr. A 760, 264-270 (1997).

$8 \mathrm{Holm}, \mathrm{D}$. K. et al. Molecular and chemical characterization of the biosynthesis of the 6-MSA derived meroterpenoid yanuthone D in Aspergillus niger. Chem. Biol. 21, 519-529 (2014).

9 Trienens, M. \& Rohlfs, M. Experimental evolution of defense against a competitive mold confers reduced sensitivity to fungal toxins but no increased resistance in Drosophila larvae. BMC Evol. Biol. 11, 1-10 (2011).

10 Hiort, J. et al. New natural products from the sponge-derived fungus Aspergillus niger J. Nat. Prod. 67, 1532-1543 (2004).

11 Nielsen, K. F., Mogensen, J. M., Johansen, M., Larsen, T. O. \& Frisvad, J. C. Review of secondary metabolites and mycotoxins from the Aspergillus niger group. Anal. Bioanal. Chem. 395, 1225-1242 (2009).

12 Tanaka, H., Wang, P.-L., Yamada, O. \& Tamura, L. T. Yellow pigments of Aspergillus niger and Asp. awamori. Part I isolation of aurasperone A and related pigments. Agric. Biol. Chem. 30, 107-113 (1966)

13 Varoglu, M. \& Crews, P. Biosynthetically diverse compounds from a saltwater culture of sponge-derived Aspergillus niger. J. Nat. Prod. 63, 41-43 (2000).

14 Gunstone, F. D., Polard, M. R., Scrimgeour, C. M. \& Vedanayagam, H. S. Fatty acids Part 50. $13 \mathrm{C}$ nuclear magnetic resonance studies of olefinic fatty acids and esters. Chem. Phys. Lipids 18, 115-129 (1977).

15 Laufer, S. \& Striegel, H. G. EP0763036B1: Annelierte pyrrolderivate und deren anwendung in der pharmazie (1995).

16 Laufer, S., Striegel, H. G. \& Dannhardt, G. US005939415A: Annellated pyrrole derivatives and their use in pharmacology (1999).

17 Nozawa, K., Nakajima, S., Kawai, K.-I. \& Udagawa, S.-I. Isolation and structures of indoloditerpenes, possible biosynthetic intermediates to the tremorgenic mycotoxin, paxilline, from Emericella striata. J. Chem. Soc. Perkin Trans. 1, 2607-2610 (1988).

18 Alfatafta, A. A., Dowd, P. F., Gloer, J. B. \& Wicklow, D. T. US005519052A: New pure carbonarin derivatives - are insecticides active against Coleoptera and Lepidoptera. (1996).

19 Alfatafta, A. A., Dowd, P. F., Gloer, J. B. \& Wicklow, D. T. US005672621A: New carbonarin compounds - useful for controlling insects (1997).

20 Priestap, H. A. New naphthopyrones from Aspergillus fonsecaeus. Tetrahedron 40, 3617-3624 (1984).

21 Campos, F. R., Barison, A., Daolio, C., Ferreira, A. G. \& Rodrigues-Fo, E. Complete 1H and $13 C$ NMR assignments of aurasperone $A$ and fonsecinone $A$, two bisnaphthopyrones produced by Aspergillus aculeatus. Magn. Reson. Chem. 43 962-965 (2005).

22 Gloer, J. B. Antiinsectan natural products from fungal sclerotia. Acc. Chem. Res. 28, 343-350 (1995).

23 Darbyshir, H. L., van de Vondervoort, P. J. I. \& Dyer, P. S. Discovery of sexual reproduction in the black Aspergilli. Fungal Genet. Rep. 60 (Suppl), 290 (abstract \# 687) (2013).

Supplementary Information accompanies the paper on The Journal of Antibiotics website (http://www.nature.com/ja) 